\title{
Active regimes for particles on resource landscapes
}

\author{
L. Varga $\odot,{ }^{1}$ A. Libál $\odot,{ }^{1}$ C. J. O. Reichhardt $\odot,{ }^{2}$ and C. Reichhardt ${ }^{2}$ \\ ${ }^{1}$ Mathematics and Computer Science Department, Babeş-Bolyai University, Cluj-Napoca 400084, Romania \\ ${ }^{2}$ Theoretical Division and Center for Nonlinear Studies, Los Alamos National Laboratory, Los Alamos, New Mexico 87545, USA
}

(Received 22 August 2021; accepted 18 January 2022; published 28 January 2022)

\begin{abstract}
We introduce an active matter model composed of sterically interacting particles, which absorb resources from a substrate and move in response to resource gradients. For varied ratios of absorption rate to substrate recovery rate, we find a variety of regimes including periodic waves, partial clustering, stochastic motion, and a frozen state. If passive particles are added, they can form crystalline clusters in an active fluid. This model could be implemented using colloidal systems on feedback landscapes and can provide a soft matter realization of excitable media and ecological systems.
\end{abstract}

DOI: 10.1103/PhysRevResearch.4.013061

Active matter exhibits self-mobility [1-3], which can appear in biological $[4,5]$, social [6,7], robotic [8,9], and soft matter systems $[10,11]$. For active matter composed of particles, the motility can be modeled as a motor force providing run-and-tumble or driven diffusive propulsion [1-3], and additional dynamics can be included that induce different types of flocking behaviors $[12,13]$. Active particle assemblies exhibit a variety of phenomena that are absent in Brownian systems, such as the motility-induced phase separation or clustering that can arise even when all the pairwise particle-particle interactions are repulsive [2,10,11,14,15], as well as active jamming [16,17]. In certain biological active matter systems, the motion can be affected by local or global gradients in the environment, producing effects such as chemotaxis or drift of particles in certain directions $[4,18]$. When the attractive or repulsive substance generating the chemotactic behavior behaves diffusively, the chemotactic drift can be described by the Keller-Segal equations [19]. If the directed motion results from substrate-bound chemicals that do not diffuse, the behavior is termed haptotaxis [20,21]. There are also numerous examples of active particles that are coupled to complex environments [2], leading to phenomena such as active matter ratchets $[22,23]$, trapping or substrate-induced clustering [2,24-26], or topotaxis, where spatial gradients in the landscape generate directed motion $[27,28]$.

It is also possible for activity to arise not from a motor force carried by each particle, but instead from forces induced by a substrate. Such situations can arise in time dependent environments $[7,29]$, particles coupled to excitable media [30], and colloids on feedback substrates [31]. Active particles in these systems may perform simple steepest descent along a gradient instead of undergoing driven diffusive or run-and-tumble

Published by the American Physical Society under the terms of the Creative Commons Attribution 4.0 International license. Further distribution of this work must maintain attribution to the author(s) and the published article's title, journal citation, and DOI. motion [32]. The substrate could have a time-independent configuration; alternatively, it can evolve according to its own dynamics and/or in response to the behavior of the active particles. For example, in an autochemotactic system, an organism secretes a chemical and then reacts chemotactically to it [33]. This can produce nonreciprocal interactions between organisms that are mediated by the chemotactic field [34]. Recently, Wang et al. [9] introduced an ecology-inspired active matter system of robots interacting with a resource substrate where the robots consume the resources and are attracted to regions with the highest resource concentration. This system exhibited numerous regimes such as crystalline, liquid, glass, and jammed states.

Here we propose a soft matter active system for an assembly of sterically repulsive particles that couple to a nondiffusive resource landscape. The particle motion is governed by local gradients of the resource concentration. When a single particle sits over a group of resource sites, it experiences a net force directed towards the sites containing the highest resource levels. Collisions between adjacent particles cause localized pinning-depinning transitions that make the behavior distinct from that found in a reaction-diffusion system. Resources are depleted from sites occupied by the particle at a fixed rate, while all sites in the system recover resource levels at a different fixed rate up to a maximum resource value.

We find that for high absorption rates and low recovery rates, particle motion occurs in periodic bursts of activity via the propagation of waves through the system. Waves also propagate through reaction-diffusion patterns found in excitable media $[30,35,36]$, but in our system, there is no diffusion. As the recovery rate is increased, the pulse frequency increases until a transition occurs to a continuously fluctuating or chaotic fluid state. When the recovery rate is larger than the absorption rate, the particles become frozen into place since a resource gradient never develops. For some rate combinations, the particles form a partially clustered state due to collisions of oppositely propagating waves. When we add a second species of passive particles that do not couple to the substrate but only interact sterically with the active particles, we find a 
crystallization of the passive particles within the fluctuating fluid regime similar to the motility induced phase separation observed for active particles with motor forces.

Our system could be realized using colloids interacting with an optical substrate in the presence of feedback mechanisms [31,37] or colloids coupled to an excitable medium where the presence of a colloid locally alters the diffusion rate in the medium. Our results indicate that soft active matter could be used to explore excitable media and ecologically inspired systems.

\section{SIMULATION AND SYSTEM}

We consider a two-dimensional system of size $L_{x} \times L_{y}$ with $L_{x}=100$ and $L_{y}=200$ containing $N_{p}=3500$ particles of diameter $d=1.0$ with steric repulsive interactions. The particle area coverage is $\phi=\pi d^{2} /\left(4 L_{x} L_{y}\right)=0.549$, well below the crystallization density of $\phi_{J}=0.9$. We are considering monodisperse disks, so the crystallization or jamming occurs at a higher density than the jamming observed for bidisperse disks with a radius ratio of 1:1.4, where jamming occurs at $\phi_{J}=0.84$ [38]. The particles also couple to a substrate consisting of a fine mesh of $N_{g}=80000$ grid sites, each of size $l_{g} \times l_{g}$ with $l_{g}=0.5$. A given grid site interacts with at most one particle at a time. Defining $g_{c}^{i}(t)$ to be the grid site closest to the center of particle $i$ at time $t$, the "occluded sites" that exert an attractive force on the particle are defined to be the eight nearest neighbors of $g_{c}^{i}(t)$ (including diagonal nearest neighbors) and the four linear next-nearest neighbors of $g_{c}^{i}(t)$. The overdamped equation of motion of particle $i$ is given by

$$
\eta \mathbf{v}_{i}=\mathbf{F}_{i}^{\mathrm{tot}}=\mathbf{F}_{i}^{p p}+\mathbf{F}_{i}^{\mathrm{g}},
$$

where $\mathbf{v}_{i}=d \mathbf{r}_{i} / d t$ is the velocity of particle $i$ and $\eta$ is the damping constant, which is set to unity. Note that the particles have no diffusive motion but respond only to gradients in the substrate and to steric interactions with other particles.

The particle-particle interaction force $\mathbf{F}_{i}^{p p}=\sum_{j=1}^{N_{p}} k(d-$ $\left.r_{i j}\right) \Theta\left(d-r_{i j}\right) \hat{\mathbf{r}}_{i j}$ arises from harmonic repulsion with spring constant $k=20, \quad r_{i j}=\left|\mathbf{r}_{i}-\mathbf{r}_{j}\right|$, and $\hat{\mathbf{r}}_{i j}=\left(\mathbf{r}_{i}-\mathbf{r}_{j}\right) / r_{i j}$, where $\Theta$ is the Heaviside step function. The attractive substrate force is summed over the 12 grid sites occluded by particle $i, \mathbf{F}_{i}^{g}=-\sum_{k=1}^{12} S_{g}^{k} \hat{\mathbf{r}}_{i k}$, where $\hat{\mathbf{r}}_{i k}=\left(\mathbf{r}_{i}-\mathbf{r}_{k}\right) / r_{i k}, r_{i k}=$ $\left|\mathbf{r}_{i}-\mathbf{r}_{k}\right|$, and $\mathbf{r}_{k}$ is the center of grid site $k$. The attraction is proportional to the resource level $S_{g}^{k}(t)$ available at grid site $k$ at time t, which ranges from the completely depleted value $S_{g}^{k}=0$ to the saturated maximum value $S_{g}^{k}=1$. If all sites occluded by a particle have the same value of $S_{g}$, the attractive forces cancel and the particle does not move. The evolution of the resource level at grid site $l$ is $S_{g}^{l}(t+\Delta t)=$ $S_{g}^{l}(t)-O_{g}^{l} r_{\mathrm{abs}}+r_{\mathrm{rec}}$, where $r_{\mathrm{abs}}$ is the rate at which a particle absorbs resources from the grid site and $O_{g}^{l}=1(0)$ if the grid site is occluded (unoccupied). All grid sites recover resources at rate $r_{\text {rec }}$, but the value of $S_{g}^{l}$ is not allowed to exceed $S_{g}^{l}=1$. Importantly, there is no diffusion of the resources themselves, unlike in typical diffusion-reaction models. For initialization, $S_{g}^{l}(0)$ for each site is set randomly to values between 0 and 1 , while the particles are placed at randomly chosen locations with particle overlap forbidden. To characterize the behavior,
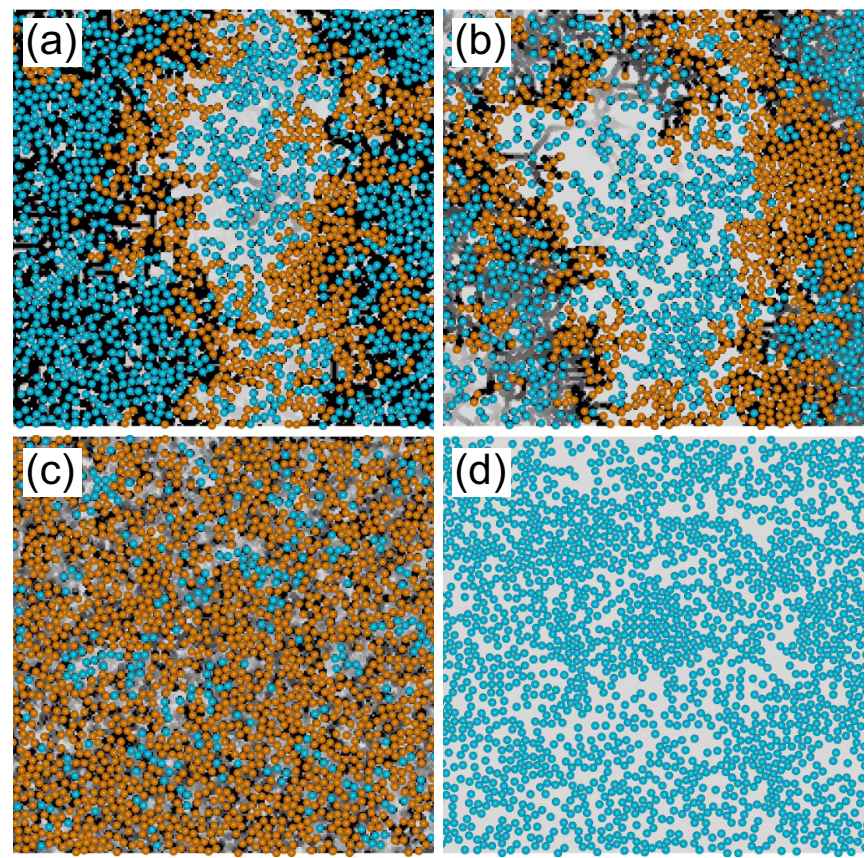

FIG. 1. Simulation snapshots of a $100 \times 100$ portion of the sample showing moving (orange) and stationary (blue) particles on a resource field with resource values ranging from $S_{g}=0$ (black) to $S_{g}=1$ (white). (a) Particles move in propagating waves for high absorption rate $r_{\text {abs }}=8.5 \times 10^{-3}$ and low recovery rate $r_{\text {rec }}=5 \times$ $10^{-5}$. (b) For higher recovery rate $r_{\text {rec }}=5 \times 10^{-4}$ at $r_{\text {abs }}=8.5 \times$ $10^{-3}$, pulses of motion appear more frequently and partial clustering of the moving particles occurs. (c) When $r_{\text {rec }}$ is comparable to $r_{\mathrm{abs}}$, a continuously moving or fluid regime appears, as shown for $r_{\mathrm{abs}}=2.5 \times 10^{-3}$ and $r_{\mathrm{rec}}=7 \times 10^{-4}$. (d) The system is frozen when $r_{\text {rec }}>r_{\text {abs }}$, as shown for $r_{\text {abs }}=4 \times 10^{-4}$ and $r_{\text {rec }}=8 \times 10^{-4}$. Movies of these states appear in the Supplemental Material [39].

we measure the fraction of particles that are moving at each instant, $M=N_{p}^{-1} \sum_{i}^{N_{p}} \Theta\left(\left|\mathbf{F}_{i}^{\mathrm{tot}}\right|-0.01\right)$, its time average $\langle M\rangle$, and its standard deviation $\sigma_{M}$. We also measure $C_{l}$, the fraction of particles belonging to the largest cluster in the system, where particles that are in contact with each other are defined as belong to a given cluster, similar to the measurement performed in Ref. [24].

\section{RESULTS}

In Fig. 1 we show snapshots of a portion of the system in different dynamic regimes. At high absorption rates and low recovery rates, illustrated in Fig. 1(a) at $r_{\mathrm{abs}}=8.5 \times 10^{-3}$ and $r_{\text {rec }}=5 \times 10^{-5}$, long intervals of no motion are interspersed with bursts of motion in the form of a front or wave that traverses the system and leaves behind a region of depleted resource sites. After each wave dissipates, the system returns to a nonmoving state until the grid sites have recovered enough resources to reactivate the particles. If we increase $r_{\text {rec }}$ while holding $r_{\text {abs }}$ fixed, the time between pulses of motion is reduced, the propagating fronts become wider, and we find a partial clustering effect as shown in Fig. 1(b) at $r_{\text {abs }}=$ $8.5 \times 10^{-3}$ and $r_{\text {rec }}=5 \times 10^{-4}$. As the recovery rate is further increased, the pulse frequency increases until a transition 


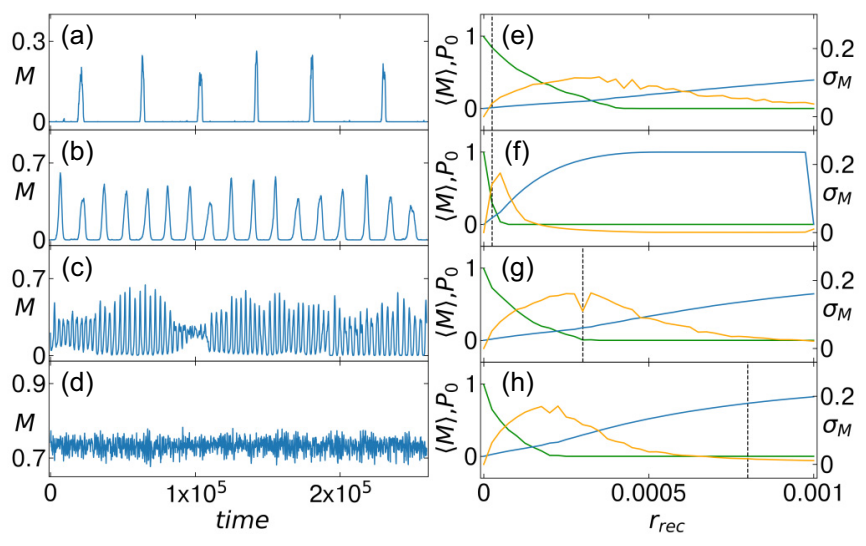

FIG. 2. Left column: The fraction of moving particles $M$ versus time in simulation steps. (a) In regime $\mathrm{I}$ at $r_{\mathrm{rec}}=2.5 \times 10^{-5}$ and $r_{\text {abs }}=0.01$, there are slow periodic oscillations in $M$. (b) At $r_{\text {rec }}=2.5 \times 10^{-5}$ and $r_{\text {abs }}=0.001$, we find regime I motion but with shorter intervals between bursts. (c) At $r_{\text {rec }}=3 \times 10^{-4}$ and $r_{\text {abs }}=$ 0.006 , the system is in regime II with rapid periodic motion. (d) For $r_{\text {rec }}=8 \times 10^{-4}$ and $r_{\text {abs }}=0.004$, we find regime III continuous motion. Right column: The time averaged fraction of moving particles $\langle M\rangle$ (blue), its standard deviation $\sigma_{M}$ (orange), and the fraction of time $P_{0}$ during which the motion is zero (green) versus recovery rate $r_{\text {rec }}$ at absorption rates of $r_{\text {abs }}=$ (e) 0.01 , (f) 0.001 , (g) 0.006, and (h) 0.004 matching the rates shown in panels (a) through (d). Dashed lines in the right column indicate the value of $r_{\text {rec }}$ illustrated in the left column.

occurs to a state in which most particles are moving most of the time, creating a fluctuating fluid as illustrated in Fig. 1(c) for $r_{\mathrm{abs}}=2.5 \times 10^{-3}$ and $r_{\mathrm{rec}}=7 \times 10^{-4}$. For high recovery and low absorption rates, the system becomes trapped in a frozen state, as shown in Fig. 1(d) at $r_{\text {abs }}=4 \times 10^{-4}$ and $r_{\text {rec }}=8 \times 10^{-4}$. The overall behavior can be described in ecological terminology by allowing the resources to represent food. Oscillations occur when there is not enough food to support continuous motion; instead, a food accumulation period is required to induce movement. Once the food has recovered, at least one particle moves and collides with another particle, triggering the wave of motion. After the wave has propagated through the system, the food is depleted again and motion does not resume until the food has been replenished. In the fluctuating state of Fig. 1(c), when food is depleted at a local grid site, the particle can immediately find sufficient food at neighboring grid sites, permitting the particle to maintain nearly constant motion. When the food is extremely plentiful, a particle always finds more than enough food at its current position, so it does not need to move.

In Fig. 2(a) we plot the fraction of moving particles $M$ versus time for a system with propagating waves at $r_{\text {rec }}=$ $2.5 \times 10^{-5}$ and $r_{\mathrm{abs}}=0.01$, where long intervals of no activity are interspersed with sharp bursts of motion. For the same system with a lower $r_{\mathrm{abs}}=0.001$ in Fig. 2(b), $M$ still has windows of close to zero activity, but the interval between activity bursts is almost 3.5 times shorter. In Fig. 2(c), where $r_{\text {rec }}=$ $3 \times 10^{-4}$ and $r_{\text {abs }}=0.006$, we find a more rapid oscillating behavior associated with the propagation of multiple fronts though the system with slightly different periods, producing beat-like patterns in $M$. For $r_{\text {rec }}=8 \times 10^{-4}$ and $r_{\text {abs }}=0.004$ in Fig. 2(d), the motion is continuous and chaotic, similar to what is shown in Fig. 1(c). In the frozen regime, such as that illustrated in Fig. 1(d), we find $M=0$ apart from a brief initial transient.

In Figs. 2(e)-2(h) we plot the time averaged fraction of moving particles $\langle M\rangle$, its standard deviation $\sigma_{M}$, and the fraction of time $P_{0}$ during which $M=0$ versus $r_{\text {rec }}$ at $r_{\text {abs }}=0.01$, $0.001,0.006$, and 0.004 , respectively. We use these measures to delineate the dynamic regimes of the system. In regime I, intermittent or periodic burst behavior of the type shown in Figs. 2(a) and 2(b) appears. Here, a single moving front of particles activates adjacent particles by pushing them forward, creating a wave-like excitation. The motion depletes the resources of the substrate, so the particles become motionless after the wave passes, and a finite recovery time is necessary before the substrate can support particle motion again. A long recovery time appears as large values of $P_{0}$ for low $r_{\text {rec }}$. Throughout regime I, $\langle M\rangle$ increases with increasing $r_{\text {rec }}$, but it exhibits large variations as indicated by the increase in $\sigma_{M}$ with increasing $r_{\text {rec }}$. Regime II is defined to occur when $P_{0}<0.5$ but the motion is still periodic. In this regime the waiting time for the substrate recovery is reduced, and motion initiates at multiple points, producing multiple simultaneously propagating fronts. The result is very rapid oscillations in $M$ and the appearance of a peak in $\sigma_{M}$. At even higher $r_{\text {rec }}$, there is a transition to regime III, consisting of continuous or chaotic motion. Here, $\sigma_{M}$ decreases with increasing $r_{\text {rec }}$ as the intermittent nature of the motion disappears, while $P_{0}$ drops and $\langle M\rangle$ becomes large. When $r_{\text {rec }}$ is increased further, there is a sudden transition to the frozen state, termed regime IV, where the recovery is sufficiently rapid that all grid sites remain saturated to $S_{g}=1$ and gradients in $S_{g}$, required to produce motion of the particles, do not develop. Figure 2(f) shows that in a sample with $r_{\mathrm{abs}}=0.001$, the frozen regime IV appears at $r_{\text {rec }}=0.001$, where $\langle M\rangle$ drops abruptly to zero. The oscillating motion in regimes I and II and the transition to the chaotic regime III motion bear similarities to the oscillations found in reaction-diffusion systems $[30,35,36]$; however, due to the lack of diffusion in our system, the fronts are correlated with translating motion of the particles rather than with reaction activity.

By conducting a series of simulations in which we measure $\langle M\rangle$ and $P_{0}$, we construct a dynamic regime diagram as a function of $r_{\text {abs }}$ versus $r_{\text {rec }}$ as shown in Fig. 3(a). The transition from I to II appears at $r_{\mathrm{abs}}=75 r_{\text {rec }}$, the II-III crossover occurs when $r_{\mathrm{abs}} \approx 7.5 r_{\mathrm{rec}}$, and the transition from III to IV falls at $r_{\text {abs }}=r_{\text {rec. }}$. In Fig. 3(b), we plot a heat map of $C_{l}$, the fraction of particles in the largest cluster, as a function of $r_{\mathrm{abs}}$ versus $r_{\text {rec }}$ for the same system. The partial clustering state appears in regime II close to the peak value of $\sigma_{M}$, indicated by a black line in Fig. 3(a). In general, clustering occurs when the pulses of motion begin to overlap. In regime III, the motion becomes too rapid for cluster formation to be possible. We note that the regime diagram indicates that the ratio of $r_{\mathrm{abs}}$ and $r_{\text {rec }}$ is important for determining the behavior, suggesting that the system could be described in terms of this ratio; however, when passive particles are added, as shown below, the behavior no longer follows a simple ratio. Thus it is useful to plot the results as a function of both $r_{\mathrm{abs}}$ and $r_{\mathrm{rec}}$. 

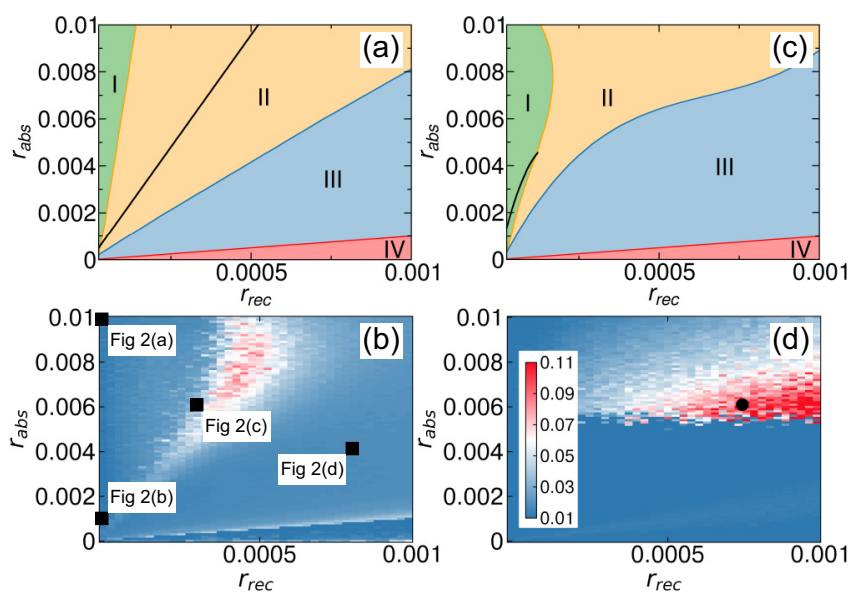

FIG. 3. (a) Dynamic regime diagram as a function of $r_{\text {abs }}$ versus $r_{\text {rec }}$ showing regimes I (periodic bursts, green), II (oscillations, yellow), III (continuous motion, blue), and IV (frozen, red). The black line indicates the location of the peak in $\sigma_{M}$. (b) Heat map of the fraction $C_{l}$ of particles in the largest cluster for the same system. Cluster size is maximized in regime II near the location of the peak in $\sigma_{M}$. (c) Dynamic regime diagram as a function of $r_{\mathrm{abs}}$ vs $r_{\mathrm{rec}}$ in a system where half of the particles are passive and do not interact with the substrate. (d) The corresponding heat map of $C_{l}$ showing that crystallization now occurs in regime III, where the passive particles form triangular crystallites as illustrated in Fig. 5.

The time series $M(t)$ of the fluctuations in the fraction of moving particles can also be characterized using the power spectrum, $S(\omega)=\left|\int M(t) \exp (-i \omega) d t\right|^{2}$. When the motion is periodic, $S(\omega)$ contains a clear peak at the dominant periodic frequency of the system. In Figs. 4(a) and 4(b) we plot the power spectra for the system in Figs. 2(a) and 2(b) in regime
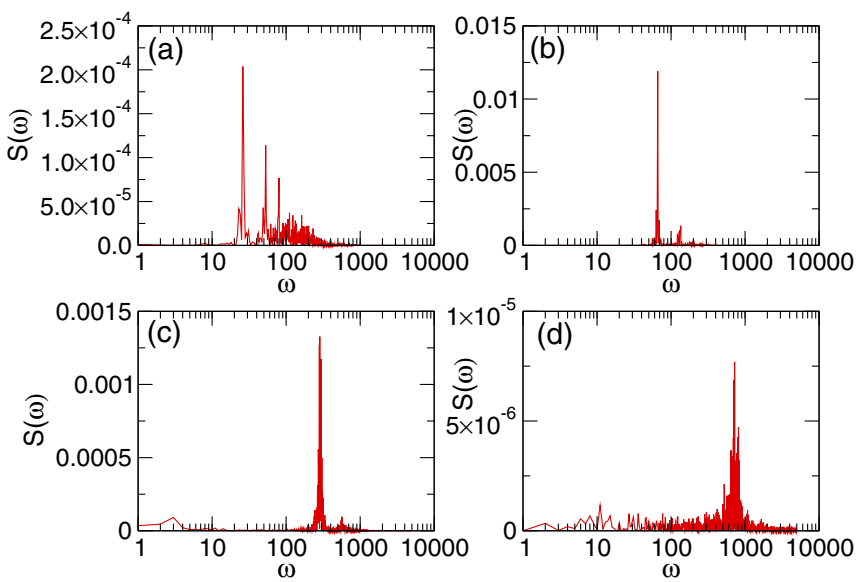

FIG. 4. The power spectra $S(\omega)$ for the time series illustrated in Fig. 3. (a) Regime I at $r_{\text {rec }}=2.5 \times 10^{-5}$ and $r_{\text {abs }}=0.01$. (b) Regime I at $r_{\text {rec }}=2.5 \times 10^{-5}$ and $r_{\mathrm{abs}}=0.001$. (c) Regime II at $r_{\mathrm{rec}}=$ $3 \times 10^{-4}$ and $r_{\mathrm{abs}}=0.006$. (d) Regime III at $r_{\text {rec }}=8 \times 10^{-4}$ and $r_{\text {abs }}=0.004$. In regimes I and II for panels (a), (b), and (c), the periodic pulses of activity produce a peak in $S(\omega)$, which shifts to higher frequencies as the oscillations become more rapid. In the continuous flow regime III found in panel (d), $S(\omega)$ has a white noise signature with almost no peak.
I, where the dynamics occurs in periodic pulses. In both cases there is a sharp peak correlated with the pulse frequency, and in Fig. 4(b), the peak shifts to higher frequency when the pulses become more rapid. The magnitude of the peak is considerably larger in Fig. 4(b), indicating that the regularity of the pulses is greater. The spectra from the signals in Figs. 2(c) and 2(d) appear in Figs. 4(c) and 4(d). For regime II in Fig. 4(c), there is still a sharp peak at even higher frequencies compared to the regime I motion since the oscillations are now very rapid. In the continuous flow regime of Fig. 4(d), the power spectrum is white and the noise power is spread among a broad range of frequencies. There is only a very weak peak at the higher frequencies that is two orders of magnitude smaller than the peaks found in regimes I and II.

It is also possible to quantify the activity using the distribution of individual particle velocities (not shown). In the periodic pulse regime, the distribution has a peak at zero activity and a long tail or second peak at finite activity, while in the continuously moving regime, the velocities obey a Gaussian distribution centered at a finite velocity and there is no weight at zero velocity.

We have also considered a system in which half of the particles are passive and interact only with other particles but not with the substrate. As shown in the dynamic regime diagram of Fig. 3(c), we find the same four regimes that appeared in the system containing only active particles; however, the range of parameters over which regime III extends is expanded. In regime II there is no partial clustering; instead, a phase separated crystallization state appears in regime III, as illustrated in the heat map of $C_{l}$ in Fig. 3(d). In the crystallized state, the passive particles form dense clusters with triangular ordering. The behavior at early and later times is illustrated in Figs. 5(a) and 5(b) for the system in Fig. 3(d) at $r_{\mathrm{abs}}=0.006$ and $r_{\text {rec }}=0.00075$. At early times, the particles are in a uniform fluid state, but at later times, dense crystalline clusters of passive particles coexist with a fluid of active particles, similar to what is observed in motility induced phase separation systems $[2,3,10,11,15]$. The crystallization occurs when $r_{\text {rec }}$ is high and $r_{\text {abs }}$ is at intermediate values. For lower $r_{\mathrm{abs}}$, the active particles move rapidly from site to site with a mostly Brownian characteristic, while for high $r_{\mathrm{abs}}$, the active particles move only in bursts separated by long time intervals, so there is not enough activity to generate the phase separation. At intermediate values of $r_{\text {abs }}$, the substrate gradients are maximized, generating the longest persistent intervals of active particle motion, and producing behavior similar to that found in run-and-tumble or driven diffusive systems.

\section{DISCUSSION}

Although some of our results, such as the appearance of periodic waves of motion, resemble the behavior that occurs in a reaction-diffusion system, there are a number of key differences. Ordinary reaction-diffusion models, such as the Belousov-Zhabotinsky reaction, do not contain translating particles, but involve only diffusing substances. In models of bacteria or other active particles undergoing autochemotaxis, both the chemotactic chemicals and the particles themselves experience diffusive behavior [40]. Models of haptotaxis, where cell motion is induced by an adhesion gradient that is 


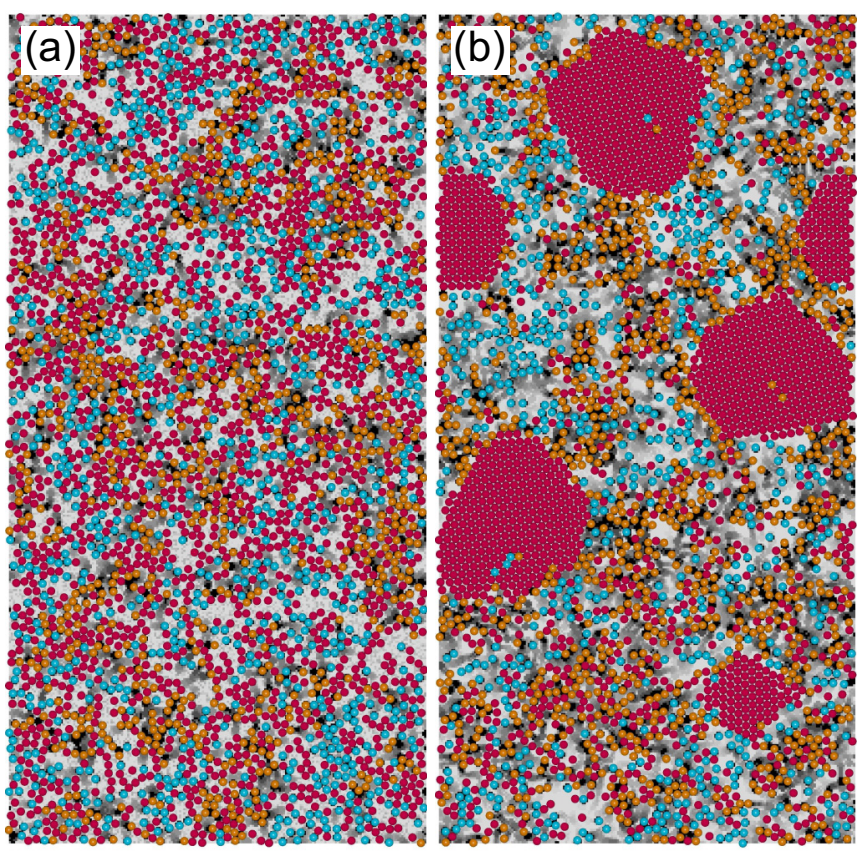

FIG. 5. Simulation snapshots of the entire sample showing moving (orange) and stationary (light blue) active particles along with passive (red) particles on a resource field with values ranging from $S_{g}=0$ (black) to $S_{g}=1$ (white) in a sample with $r_{\text {abs }}=0.006$ and $r_{\text {rec }}=0.00075$. (a) At early times, the system forms a regime III uniform fluctuating state. (b) At later times, the passive particles are pushed together into crystalline clusters. Movies of these states appear in the Supplemental Material [39].

localized in the extracellular matrix [41], involve chemicals that are affixed to a substrate and do not diffuse. Such haptotaxis models typically assume a static substrate [21]. In an autohaptotactic process, the adhesion gradient is modified by the cell as it travels. For example, realization of an artificial system that can modify its own environment was achieved using vesicles on a lipid bilayer [42]. Cells can also metabolize or destroy a chemical on the substrate and then follow the resulting self-generated gradient in a method of motion that may be important for a number of biological processes [43-48]. Studies of trail-mediated interactions, in which the organisms emit chemicals that diffuse very slowly relative to the motion of the organism, include diffusional motion of the organisms themselves [49,50], which is absent in our model. Another method of generating trail-mediated interactions using time-delayed interactions of a particle with its own previous position also incorporated diffusion of the particles [51]. A model that specifically considers consumption of a resource, namely oxygen, by individual cells, included diffusion of that resource [52]. In our system, there is no diffusive term in the evolution of either the particles or the resources; instead, the particles consume the resources and move in response to the resulting local resource gradients, while also interacting sterically with other particles. The lack of diffusion and the importance of the collective steric interactions between the particles makes our system significantly different from standard models. In particular, unlike the model in [33], our system has no long-time diffusive limit that can be reached by either exhausting the resource supply or flattening the resource gradient; instead, the particles become frozen in these limits. The particles are inherently out of equilibrium and can experience nonreciprocal forces, which are one of the sources of the clustering and the phase separation.

Another question is whether there are true phase transitions among the different regimes we observe. Since our system is inherently non-equilibrium, this is not straightforward to address. It is possible that some regimes are on either side of a non-equilibrium phase transition, while other regimes are merely crossovers. For example, the continuously moving phase could be bounded by an absorbing phase transition, with the fluctuating state represented by the continuously moving behavior and the absorbed state represented by the oscillating state. If this is the case, there could be a divergence in the amount of time the system remains active on the absorbing side of the transition [53-55].

Possible experimental realizations of this system include robotic systems programmed to couple to a reactive landscape [9,56], colloidal particles interacting with optical feedback arrays $[31,57]$, or even self-propelled chemically active colloids coupled to a reaction-diffusion surface where the colloids exhibit propulsion along chemical gradients. Additional possibilities include colloidal particles coupled to mobile cells on a substrate, similar to what was considered in Refs. [58-61]. Other variations of this system would be possible including coupling bacteria or biological agents to a substrate where food or resources are added at a continuous rate.

We have proposed an active matter system in which particles interact with a resource landscape of grid sites that impart forces to the particles. Particles are attracted to sites with the most resources, and simultaneously experience steric repulsion from other particles. Sites underneath particles have their resources depleted at a fixed absorption rate, while all sites recover their resources at a different fixed recovery rate up to a maximum value. When the absorption rate is much larger than the recovery rate, we find periodic pulses of propagating waves of translating particles. As the recovery rate increases, the time between pulses decreases until there is a transition to a fluid-like state with continuous motion. When the recovery rate is greater than or equal to the absorption rate, the system becomes trapped in a frozen state. When we introduce a second species of passive particles that do not couple to the substrate but interact sterically with all other particles, crystalline clusters of passive particles can form within the fluid state. Our system could be realized using colloidal particles interacting with an optical feedback light substrate, reactive excitable media, or robotic assemblies with an active substrate. This system could also be used to create soft matter versions of ecological models and excitable media.

\section{ACKNOWLEDGMENTS}

We gratefully acknowledge the support of the U.S. Department of Energy through the LANL/LDRD program for this work. This work was supported by the US Department of Energy through the Los Alamos National Laboratory. Los Alamos National Laboratory is operated by Triad National Security, LLC, for the National Nuclear Security Administration of the U. S. Department of Energy (Contract No. 
892333218NCA000001). The work of L.V. and A.L. was supported by a Grant of the Romanian Ministry of Education and Research, CNCS - UEFISCDI, Project No. PN-III-P4-IDPCE-2020-1301, within PNCDI III.
[1] M. C. Marchetti, J. F. Joanny, S. Ramaswamy, T. B. Liverpool, J. Prost, M. Rao, and R. A. Simha, Hydrodynamics of soft active matter, Rev. Mod. Phys. 85, 1143 (2013).

[2] M. E. Cates and J. Tailleur, Motility-induced phase separation, Annu. Rev. Condens. Matter Phys. 6, 219 (2015).

[3] C. Bechinger, R. Di Leonardo, H. Löwen, C. Reichhardt, G. Volpe, and G. Volpe, Active particles in complex and crowded environments, Rev. Mod. Phys. 88, 045006 (2016).

[4] H. C. Berg, Random Walks in Biology, Vol. 4 (Princeton University Press, Princeton, NJ, 1983).

[5] D. Dell'Arciprete, M. L. Blow, A. T. Brown, F. D. C. Farrell, J. S. Lintuvuori, A. F. McVey, D. Marenduzzo, and W. C. K. Poon, A growing bacterial colony in two dimensions as an active nematic, Nat. Commun. 9, 4190 (2018).

[6] D. Helbing, Traffic and related self-driven many-particle systems, Rev. Mod. Phys. 73, 1067 (2001).

[7] I. D. Couzin, J. Krause, N. R. Franks, and S. A. Levin, Effective leadership and decision-making in animal groups on the move, Nature (London) 433, 513 (2005).

[8] A. Deblais, T. Barois, T. Guerin, P. H. Delville, R. Vaudaine, J. S. Lintuvuori, J. F. Boudet, J. C. Baret, and H. Kellay, Boundaries Control Collective Dynamics of Inertial Self-Propelled Robots, Phys. Rev. Lett. 120, 188002 (2018).

[9] G. Wang, T. V. Phan, S. Li, M. Wombacher, J. Qu, Y. Peng, G. Chen, D. I. Goldman, S. A. Levin, R. H. Austin, and L. Liu, Emergent Field-Driven Robot Swarm States, Phys. Rev. Lett. 126, 108002 (2021).

[10] J. Palacci, S. Sacanna, A. P. Steinberg, D. J. Pine, and P. M. Chaikin, Living crystals of light-activated colloidal surfers, Science 339, 936 (2013).

[11] I. Buttinoni, J. Bialké, F. Kümmel, H. Löwen, C. Bechinger, and T. Speck, Dynamical Clustering and Phase Separation in Suspensions of Self-Propelled Colloidal Particles, Phys. Rev. Lett. 110, 238301 (2013).

[12] T. Vicsek and A. Zaferis, Collective motion, Phys. Rep. 517, 71 (2012).

[13] L. Barberis and F. Peruani, Large-Scale Patterns in a Minimal Cognitive Flocking Model: Incidental Leaders, Nematic Patterns, and Aggregates, Phys. Rev. Lett. 117, 248001 (2016).

[14] Y. Fily and M. C. Marchetti, Athermal Phase Separation of SelfPropelled Particles with No Alignment, Phys. Rev. Lett. 108, 235702 (2012).

[15] G. S. Redner, M. F. Hagan, and A. Baskaran, Structure and Dynamics of a Phase-Separating Active Colloidal Fluid, Phys. Rev. Lett. 110, 055701 (2013).

[16] S. Henkes, Y. Fily, and M. C. Marchetti, Active jamming: Self-propelled soft particles at high density, Phys. Rev. E 84, 040301(R) (2011).

[17] R. Mandal, P. J. Bhuyan, P. Chaudhuri, C. Dasgupta, and M. Rao, Extreme active matter at high densities, Nat. Commun. 11, 2581 (2020).

[18] H. Stark, Artificial chemotaxis of self-phoretic active colloids: Collective behavior, Acc. Chem. Res. 51, 2681 (2018).
[19] E. F. Keller and L. A. Segel, Initiation of slime mold aggregation viewed as an instability, J. Theor. Biol. 26, 399 (1970).

[20] S. B. Carter, Haptotaxis and mechanism of cell motility, Nature (London) 213, 256 (1967).

[21] S. G. Ricoult, T. E. Kennedy, and D. Juncker, Substratebound protein gradients to study haptotaxis, Front. Bioeng. Biotechnol. 3, 40 (2015).

[22] P. Galajda, J. Keymer, P. Chaikin, and R. Austin, A wall of funnels concentrates swimming bacteria, J. Bacteriol. 189, 8704 (2007).

[23] C. J. Olson Reichhardt and C. Reichhardt, Ratchet effects in active matter systems, Ann. Rev. Condens. Matter Phys. 8, 51 (2017).

[24] C. Reichhardt and C. J. Olson Reichhardt, Active matter transport and jamming on disordered landscapes, Phys. Rev. E 90, 012701 (2014).

[25] A. Morin, N. Desreumaux, J.-B. Caussin, and D. Bartolo, Distortion and destruction of colloidal flocks in disordered environments, Nat. Phys. 13, 63 (2017).

[26] T. Bhattacharjee and S. S. Dutta, Bacterial hopping and trapping in porous media, Nat. Commun. 10, 2075 (2019).

[27] K. Schakenraad, L. Ravazzano, N. Sarkar, J. A. J. Wondergem, R. M. H. Merks, and L. Giomi, Topotaxis of active Brownian particles, Phys. Rev. E 101, 032602 (2020).

[28] M. Vergassola, E. Villermaux, and B. I. Shraiman, 'infotaxis' as a strategy for searching without gradients, Nature (London) 445, 406 (2007).

[29] M. Cenzer and L. K. M'Gonigle, Local adaptation in dispersal in multi-resource landscapes, Evolution 73, 648 (2019).

[30] V. S. Zykov and E. Bodenschatz, Wave propagation in inhomogeneous excitable media, Ann. Rev. Condens. Matter Phys. 9, 435 (2018).

[31] T. Bäuerle, A. Fischer, T. Speck, and C. Bechinger, Selforganization of active particles by quorum sensing rules, Nat. Commun. 9, 3232 (2018).

[32] A. Dhariwal, G. S. Sukhatme, and A. A. G Requicha, in Proceedings of IEEE International Conference on Robotics and Automation, ICRA '04 (IEEE, 2004), pp. 1436-1443.

[33] A. Sengupta, S. van Teeffelen, and H. Löwen, Dynamics of a microorganism moving by chemotaxis in its own secretion, Phys. Rev. E 80, 031122 (2009).

[34] V. Ouazan-Reboul, J. Agudo-Canalejo, and R. Golestanian, Non-equilibrium phase separation in mixtures of catalytically active particles: Size dispersity and screening effects, Eur. Phys. J. E 44, 113 (2021).

[35] A. N. Zaikin and A. M. Zhabotinsky, Concentration wave propagation in two-dimensional liquid-phase self-oscillating system, Nature (London) 225, 535 (1970).

[36] T. Sakurai, E. Mihaliuk, F. Chirila, and K. Showalter, Design and control of wave propagation patterns in excitable media, Science 296, 2009 (2002). 
[37] F. A. Lavergne, H. Wendehenne, T. Baeuerle, and C. Bechinger, Group formation and cohesion of active particles with visual perception-dependent motility, Science 364, 70 (2019).

[38] C. Reichhardt and C. J. Olson Reichhardt, Aspects of jamming in two-dimensional athermal frictionless systems, Soft Matter 10, 2932 (2014).

[39] See Supplemental Material at http://link.aps.org/supplemental/ 10.1103/PhysRevResearch.4.013061 for movies of the particles interacting with the resource grid.

[40] J. Taktikos, V. Zaburdaev, and H. Stark, Modeling a selfpropelled autochemotactic walker, Phys. Rev. E 84, 041924 (2011).

[41] M. Weber, R. Hauschild, J. Schwarz, C. Moussion, I. de Vries, D. F. Legler, S. A. Luther, T. Bollenbach, and M. Sixt, Interstitial dendritic cell guidance by haptotactic chemokine gradients, Science 339, 328 (2013).

[42] J. Solon, P. Streicher, R. Richter, F. Brochard-Wyart, and P. Bassereau, Vesicles surfing on a lipid bilayer: Self-induced haptotactic motion, Proc. Natl. Acad. Sci. U.S.A. 103, 12382 (2006).

[43] L. Tweedy, O. Susanto, and R. H. Insall, Self-generated chemotactic gradients-Cells steering themselves, Curr. Opin. Cell Biol. 42, 46 (2016).

[44] C. Scherber, A. J. Aranyosi, B. Kulemann, S. P. Thayer, M. Toner, O. Iliopoulos, and D. Irimia, Epithelial cell guidance by self-generated EGF gradients, Integr. Biol. 4, 259 (2012).

[45] E. Donà, J. D. Barry, G. Valentin, C. Quirin, A. Khmelinskii, A. Kunze, S. Durdu, L. R. Newton, A. Fernandez-Minan, W. Huber et al., Directional tissue migration through a self-generated chemokine gradient, Nature (London) 503, 285 (2013).

[46] A. J. Muinonen-Martin, O. Susanto, Q. F. Zhang, E. Smethurst, W. J. Faller, D. M. Veltman, G. Kalna, C. Lindsay, D. C. Bennett, O. J. Sansom et al., Melanoma cells break down LPA to establish local gradients that drive chemotactic dispersal, PLoS Biol. 12, e1001966 (2014).

[47] L. Tweedy, D. A. Knecht, G. M. Mackay, and R. H. Insall, Self-generated chemoattractant gradients: Attractant depletion extends the range and robustness of chemotaxis, PLoS Biol. 14, e1002404 (2016).

[48] L. Tweedy, P. A. Thomason, P. I. Paschke, K. Martin, L. M. Machesky, M. Zagnoni, and R. H. Insall, Seeing around corners: Cells solve mazes and respond at a distance using attractant breakdown, Science 369, 1075 (2020).

[49] W. T. Kranz, A. Gelimson, K. Zhao, G. C. L. Wong, and R. Golestanian, Effective Dynamics of Microorganisms That Interact with Their Own Trail, Phys. Rev. Lett. 117, 038101 (2016).

[50] W. T. Kranz and R. Golestanian, Trail-mediated self-interaction, J. Chem. Phys. 150, 214111 (2019).

[51] B. Liebchen and H. Löwen, Synthetic chemotaxis and collective behavior in active matter, Acc. Chem. Res. 51, 2982 (2018).

[52] O. Cochet-Escartin, M. Demircigil, S. Hirose, B. Allais, P. Gonzalo, I. Mikaelian, K. Funamoto, C. Anjard, and V. Calvez, Hypoxia triggers collective aerotactic migration in Dictyostelium discoideum, eLife 10, e64731 (2021).

[53] H. Hinrichsen, Non-equilibrium critical phenomena and phase transitions into absorbing states, Adv. Phys. 49, 815 (2000).

[54] L. Corté, P. M. Chaikin, J. P. Gollub, and D. J. Pine, Random organization in periodically driven systems, Nat. Phys. 4, 420 (2008).

[55] C. Reichhardt and C. J. Olson Reichhardt, Absorbing phase transitions and dynamic freezing in running active matter systems, Soft Matter 10, 7502 (2014).

[56] M. Leyman, F. Ogemark, J. Wehr, and G. Volpe, Tuning phototactic robots with sensorial delays, Phys. Rev. E 98, 052606 (2018).

[57] S. Tarama, S. U. Egelhaaf, and H. Löwen, Traveling band formation in feedback-driven colloids, Phys. Rev. E 100, 022609 (2019).

[58] D. Bi, J. H. Lopez, J. M. Schwarz, and M. L. Manning, A density-independent rigidity transition in biological tissues, Nat. Phys. 11, 1074 (2015).

[59] D. Bi, X. Yang, M. C. Marchetti, and M. L. Manning, MotilityDriven Glass and Jamming Transitions in Biological Tissues, Phys. Rev. X 6, 021011 (2016).

[60] L. Atia, D. Bi, Y. Sharma, J. A. Mitchel, B. Gweon, S. A. Koehler, S. J. DeCamp, B. Lan, J. H. Kim, R. Hirsch et al., Geometric constraints during epithelial jamming, Nat. Phys. 14, 613 (2018).

[61] V. Petrolli, M. Le Goff, M. Tadrous, K. Martens, C. Allier, O. Mandula, L. Hervé, S. Henkes, R. Sknepnek, T. Boudou, G. Cappello, and M. Balland, Confinement-Induced Transition between Wavelike Collective Cell Migration Modes, Phys. Rev. Lett. 122, 168101 ( 2019).. 